\title{
Health Care Access Barriers Bring Children to Emergency Rooms More Frequently: A Representative Survey
}

\author{
Thom Taylor, PhD, and Daria Salyakina, PhD
}

\begin{abstract}
Children may visit the emergency department (ED) regularly in part because they and their caregivers may be experiencing barriers to appropriate and timely pediatric care. However, assessing the wide range of potential barriers to access to care that children and their caregivers may experience is often a challenge. The objective of this study was to assess the barriers to pediatric health care reported by caregivers and to examine the association between those reported barriers to care with the frequency of children's ED visits in the past 12 months. Assessment of ED utilization and access to care barriers was made through a telephone interview survey conducted as part of a broader Community Health Needs Assessment in 2015. A weighted community sample of adult caregivers $(\mathrm{N}=1057)$ of children between the ages of 0-17 residing in Miami-Dade, Broward, and Palm Beach counties, Florida were contacted. This study found that multiple ED visits ( $\geq 2$ vs. 0$)$ in the past 12 months by a child were most strongly associated with access to care barriers attributed to language and culture (relative risk $[R R]=2.51$ ), trouble finding a doctor $(\mathrm{RR}=1.86)$, scheduling an appointment $(\mathrm{RR}=1.68)$, and transportation access $(\mathrm{RR}=1.73)$. These findings suggest that access to care barriers experienced by households may exacerbate the risk of a child experiencing repeated visits to the ED in a year. Findings are discussed further in the context of actionable population health management strategies to reduce risk of frequent ED utilization by children.
\end{abstract}

Keywords: barriers, health care access, emergency department, children, caregivers

\section{Introduction}

$\mathbf{T}$ HE EMERGENCY DEPARTMENT (ED) is not always the optimal source of care for children, ${ }^{1,2}$ though the challenges and risk factors for repeated ED utilization that children and their caregivers face may be diverse and extensive. ${ }^{3}$ Considerations identified previously that affect the likelihood of frequent ED visits include whether a child has a chronic condition, ${ }^{3}$ younger age of the child, ${ }^{3,4}$ as well as socioeconomic status (SES) indicators.

Specifically, in the United States of America, poorer, unemployed individuals, nonimmigrants, and black or African Americans appear to have higher utilization of EDs. ${ }^{4-6}$ The source of health insurance for a child is also often linked to frequent ED utilization. Commonly, the child is less likely to utilize the ED if he or she has private insurance relative to public forms of insurance (eg, Medicaid). ${ }^{7,8}$ Inconsistent health coverage also may lead to repeated ED utilization as lack of health insurance can result in delayed care, unfilled prescriptions, limited well-child visits, and fractured health care for children.,
Even when a child has some form of health insurance, some caregivers still may struggle to find regular sources of care if fewer and fewer providers accept the child's health insurance. Such narrowing of networks, defined as a network that is "sufficient in number and type of providers" 11 may make regular care for one's child more difficult. ${ }^{10}$ Recent evidence suggests that pediatric care may suffer from even more narrow networks than adult care. ${ }^{12}$

Moreover, even when a child is able to be seen by a provider in a particular network, attaining a timely appointment for regular care may be another hurdle. ${ }^{1}$ Still, when an appointment is available, office hours for the provider might conflict with other responsibilities of the caregiver. ${ }^{8}$ In tandem, the cost of care also may be a significant challenge for some caregivers. ${ }^{12}$ Additionally, should a child require medication, the cost of medication also may be prohibitive depending on income, co-pays, or the general expense of the medication the child may need.

Yet another challenge faced by some caregivers that does not always receive emphasis in health care is efficient

Nicklaus Children's Research Institute, Miami, Florida.

(C) Thom Taylor and Daria Salyakina 2018; Published by Mary Ann Liebert, Inc. This Open Access article is distributed under the terms of the Creative Commons License (http://creativecommons.org/licenses/by/4.0), which permits unrestricted use, distribution, and reproduction in any medium, provided the original work is properly cited. 
transportation. ${ }^{12,13}$ For example, lack of a personal vehicle may force caregivers to rely on the schedules of relatives and peers for rides, or when available in an area, public transportation schedules that are potentially challenging. As a result, more limited access to efficient and reliable transportation may inadvertently make timely access to care for a child more difficult as well. ${ }^{13}$

Finally, cultural and language challenges are an important consideration in receiving appropriate care as communication between caregivers and community health care systems can be strained because of language or culturally-related communication barriers. ${ }^{6}$ This may manifest when recent immigrant populations attempt to navigate complex health care systems in a language unfamiliar to them. ${ }^{14}$ Yet, language and culture are not equivalent, and consideration of culture in the context of health care beliefs and expected practices of physicians also may be relevant when seeking health care. ${ }^{15}$

The aforementioned challenges and potential barriers to access to timely and appropriate care for children are not widely or comprehensively assessed in a single study. This study sought to consider the wide range of potential barriers to access to care that caregivers of children may face while simultaneously accounting for macro-level indicators of risk for ED utilization. The primary objective of this study was to assess the role that the wide range of aforementioned barriers to access to care may have on past 12-month ED utilization by a child.

\section{Methods}

\section{Study design, setting, population, and sample}

A weighted sample of residents in South Florida's MiamiDade, Broward, and Palm Beach counties was surveyed on their landlines or cell phones by Professional Research Consultants Inc. (PRC) as part of a Child and Adolescent Community Health Needs Assessment (CHNA). For the telephone-administered survey, PRC assigned a caller ID number that is local to the area, made multiple attempts to reach respondents, and called at different times of day on different days of the week. The sample was stratified by county to ensure adequate representation among all strata.

At the time of the interview, for households with multiple children age $0-17$, one child was selected at random based on which had the most recent birthday, and survey questions were asked about that specific child. This produced a sample that is more representative by demographics of age and sex. Survey respondents were adults ages 18 years and older who had at least 1 child residing in the household for whom the respondent was the health care decision maker. This person is referred to as the child's caregiver. Prior to completion of the telephone interview survey caregivers provided verbal consent to participate in the CHNA telephone survey interview.

Caregivers could respond in the 2 most dominant languages in South Florida communities: Spanish and English. PRC maintains a team of native Spanish-speaking interviewers who manage calls for those respondents who speak only Spanish or are more comfortable speaking Spanish. A parallel Spanish structured survey form conveying the same sentiments in Spanish as the English form also was used when conducting the interview with Spanish-speaking caregivers. In this way, the study team was able to ensure coverage of the language considerations for a very large proportion of the population in the counties involved.

Interviews were conducted throughout 2015 with a total of $\mathrm{N}=1057$ caregivers who completed the survey interview. Data were de-identified prior to investigators receiving the data and an Institutional Review Board exempt research protocol was approved for this study.

\section{Survey content and administration}

Items in the CHNA survey inquired about health care access, health insurance, chronic conditions, and care utilization. Prior to de-identification, responses were weighted by PRC for nonresponse and further post-stratified based on sample characteristics of the child's age, sex, race/ethnicity, and household poverty status in order to align the survey responses to known population proportions using US Census Bureau population estimates.

The outcome of interest was the response to the following phone interview question: "In the past 12 months, how many times has the child gone to the hospital emergency room about (his/her) own health?" The study team was centrally interested in the question of which barriers may be associated with multiple visits to an ED in the past 12 months. Because of a very limited distribution beyond 2 visits in the past 12 months, each outcome was collapsed into 3-category multinomial responses with the levels no visits, a single visit, and multiple $(\geq 2)$ visits. Health care access difficulty items assessed in the CHNA survey are presented in Table 1. All items were binary in response choice (yes/no).

\section{Data analysis}

Limited item-level missing data $(<4 \%)$ for any 1 variable was observed. Nevertheless, to ensure complete weighted sample estimation, random forest imputation methods ${ }^{16}$

Table 1. Community Health Needs Assessment Survey Items Addressing Access to Care Difficulties

1. Was there a time in the past 12 months when this child needed medical care, but you had difficulty finding a doctor?

2. Was there a time in the past 12 months when you had difficulty getting an appointment for this child to see a doctor?

3. Was there a time in the past 12 months when this child needed to see a doctor, but could not because of the cost?

4. Was there a time in the past 12 months when a lack of transportation made it difficult or prevented this child from seeing a doctor OR kept you from making a medical appointment for this child?

5. Was there a time in the past 12 months when this child was not able to see a doctor because the office hours were not convenient?

6. Was there a time in the past 12 months when this child needed a prescription medicine, but did not get it because you could not afford it?

7. Was there a time in the past 12 months when cultural or language differences made it difficult or prevented you from getting health care for this child? 
were utilized to impute item-level nonresponse; the survey weight was incorporated in missing data imputation. Subsequently, a weighted multinomial model was fit with the focal variables of barriers to care described in Table 1 . This model also adjusted for the child's age; sex; race/ethnicity combinations as captured in the CHNA including nonHispanic white, non-Hispanic black, Hispanic, or other race/ ethnicity combination; child having a chronic health condition; the household language; and the household poverty classification based on US Department of Health and $\mathrm{Hu}-$ man Service (HHS) thresholds. ${ }^{17}$ Three HHS categories were assessed: household below $100 \%$ of the Federal poverty level, $100 \%-199 \%$ of the poverty level, and $200 \%$ and above the poverty level. Health insurance status of the child at the time of the phone interview (private, Medicaid, Medicare, other, or uninsured) was captured, as well as inconsistency in health care coverage.

Inconsistency in health care coverage was defined as any period in the child's life when he or she did not have health insurance. County of residence also was accounted for given that Miami-Dade, Broward, and Palm Beach counties vary demographically and socioeconomically in a manner similar to other large metropolitan areas in the United States.

The multinomial model also incorporated sampling and nonresponse weights to provide population-level inference. Relative risk (RR) estimates and associated $95 \%$ confidence intervals (CIs) were estimated as were the average adjusted marginal probabilities and associated 95\% CIs from the predicted model estimates.

\section{Results}

First, the weighted distribution of ED visit frequency in the sample and sample demographics were evaluated. The majority of the children in this study (66\%) had not visited the ED in the past 12 months. Approximately $18 \%$ of caregivers reported that their child had visited the ED once, and a still sizeable $16 \%$ had $\geq 2 \mathrm{ED}$ visits in the past 12 months (Table 2). Detailed weighted descriptive estimates of ED visits, demographics, and access barriers are presented in the following text and in Table 2.

\section{Patient demographics and insurance type}

Figure 1 presents the RR estimates and Figure 2 presents the corresponding average marginal probabilities for the associated RR estimates of the adjusted multinomial model. Age of the child at the time of the interview was only associated with $\geq 2$ vs. no ED visits in the past 12 months $(\mathrm{RR}=.92, P<.05)$ (Figure 1$)$. Neither sex nor race/ethnicity was significantly associated with frequency of ED visits.

Unsurprisingly, children with a chronic condition did have a higher risk of multiple $\mathrm{ED}$ visits, $(\mathrm{RR}=2.07, P<.05)$. Yet, no association between ED visits and certain socioeconomic considerations was observed (ie, poverty status). However, Spanish language use in the home was associated with a reduced likelihood of multiple $\mathrm{ED}$ visits $(\mathrm{RR}=.37, P<.05)$. Additionally, county of residence was associated with both single and multiple visits to the ED. Relative to residents of Miami-Dade County, children in Broward and Palm Beach were less likely to have a single $\mathrm{ED}$ visit $(\mathrm{RR}=.64, P<.05$ and $\mathrm{RR}=.39, P<.05$, respectively). A similar pattern was observed for children re- ported to have had multiple ED visits in the past 12 months $(\mathrm{RR}=.67, P<.05$ and $\mathrm{RR}=.33, P<.05$, respectively).

Relative to children having private insurance, having Medicaid insurance was associated with multiple ED visits $(\mathrm{RR}=2.73, P<.05)$ in the past 12 months (Figure 1). Similarly, children with Medicare had a higher risk of $\geq 2$ visits $(\mathrm{RR}=2.74, P<.05)$ (Figures 1 and 2 ). Children who did not have insurance also were twice as likely to have been in the ED multiple $(\geq 2)$ times relative to privately insured children $(\mathrm{RR}=2.04, P<.05)$. Similar effects were found with none vs. a single visit, though variability in those estimates led to less confidence about the robustness of these findings at the population level.

\section{Access to care barriers}

Out of 7 assessed barriers to health care reported by children's caregivers in the survey, 4 were statistically significantly associated with frequency of multiple ED visits. The most prominent and consistent association was found for cultural or language differences. Overall, this was not a common barrier as it was reported by only an estimated $6 \%$ (95\% CI: $4 \%-8 \%$ ) of caregivers (Table 2). Yet, it was associated with both a single $(\mathrm{RR}=2.30, P<.05)$ and multiple ED visits $(\mathrm{RR}=2.51, P<.05)$ in the past 12 months (Figure 2). Caregivers who reported experiencing cultural or language barriers to care were roughly 5.8 times more likely to report their child had $\geq 2$ ED visits (35\% vs. $6 \%$ ) and 2.5 times more likely to report a single ED visit (36\% vs. $15 \%$ ) with the child within the last 12 months when compared to caregivers who did not report experiencing this difficulty (Figure 3).

Further, difficulty finding a doctor for the child, difficulty making an appointment, and reported transportation challenges were associated with multiple $\mathrm{ED}$ visits $(\mathrm{RR}=1.86$, $P<.05 ; \mathrm{RR}=1.68, P<.05 ; \mathrm{RR}=1.73, P<.05$, respectively), but not with single ED visits (Figures 2 and 3). Those caregivers who reported that they had difficulty finding a doctor for their child in the past 12 months were 4.3 times more likely to bring their child to the ED $\geq 2$ times (26\% vs. 6\%). Similarly, caregivers who experienced difficulty making an appointment or transportation challenges were $3.6(18 \%$ vs. $5 \%)$ and $4.3(26 \%$ vs $6 \%)$ times more likely to bring their child to the ED multiple times in a 12-month period (Figure 3). The cost of a doctor, inconvenience of office hours, and the cost of medications were not associated with past 12-months ED visit frequency.

\section{Discussion}

Of central interest to the objectives of this study, the findings support the notion that demographics, comorbidities, and access to care barriers experienced in the household by caregivers may all affect ED utilization in a 12-month period. These findings highlight that caregivers who experienced barriers to appropriate access to health care for their children often may rely on the ED as a primary source of such care for their children. ${ }^{5}$

At the same time, the findings align well with previous research assessing population health dynamics and care utilization rates at a national level. For example, the Children's Hospital Association reported that upward of $37 \%$ of children in care in pediatric hospitals may be admitted to an 
Table 2. Demographics and Outcomes

\begin{tabular}{|c|c|c|c|}
\hline Variable & Level & Weighted $n$ & $\begin{array}{c}\text { Weighted } \\
\text { percent }(95 \% \text { CI) }\end{array}$ \\
\hline \multicolumn{4}{|l|}{ Outcomes } \\
\hline \multirow[t]{3}{*}{ Emergency Department Visits in Past 12 Months } & 0 Visits & 694 & $66 \%(62 \%-69 \%)$ \\
\hline & 1 Visit & 195 & $18 \%(16 \%-21 \%)$ \\
\hline & $\geq 2$ Visits & 169 & $16 \%(13 \%-19 \%)$ \\
\hline \multirow[t]{3}{*}{ Urgent Care Visits in Past 12 Months } & 0 Visits & 669 & $63 \%(60 \%-67 \%)$ \\
\hline & 1 Visit & 227 & $21 \%(19 \%-24 \%)$ \\
\hline & $\geq 2$ Visits & 162 & $15 \%(13 \%-18 \%)$ \\
\hline \multicolumn{4}{|l|}{ Demographics } \\
\hline \multirow[t]{2}{*}{ Sex of Child } & a. Male & 541 & $51 \%(48 \%-55 \%)$ \\
\hline & b. Female & 517 & $49 \%(45 \%-52 \%)$ \\
\hline \multirow[t]{4}{*}{ Race/Ethnicity of Child } & a. Hispanic & 446 & $42 \%(39 \%-45 \%)$ \\
\hline & b. NHW & 287 & $27 \%(25 \%-30 \%)$ \\
\hline & c. NHB & 254 & $24 \%(21 \%-27 \%)$ \\
\hline & d. Other & 72 & $7 \%(5 \%-9 \%)$ \\
\hline \multirow[t]{2}{*}{ Child has Chronic Condition } & a. No & 856 & $81 \%(78 \%-84 \%)$ \\
\hline & b. Yes & 202 & $19 \%(16 \%-22 \%)$ \\
\hline \multirow[t]{5}{*}{ Insurance Type Child has } & a. Private Health Insurance & 497 & $47 \%(44 \%-50 \%)$ \\
\hline & b. Medicaid & 306 & $29 \%(25 \%-32 \%)$ \\
\hline & c. Medicare & 91 & $9 \%(6 \%-11 \%)$ \\
\hline & d. Other & 79 & $8 \%(6 \%-9 \%)$ \\
\hline & e. Uninsured & 85 & $8 \%(6 \%-10 \%)$ \\
\hline \multirow{2}{*}{ Child has had Inconsistent Health Insurance Coverage } & a. No & 920 & $87 \%(84 \%-89 \%)$ \\
\hline & b. Yes & 138 & $13 \%(11 \%-16 \%)$ \\
\hline \multirow[t]{3}{*}{ Household HHS Poverty Classification } & a. $200 \%$ FPL or Higher & 601 & $57 \%(53 \%-60 \%)$ \\
\hline & b. $100 \%$ to $199 \%$ of FPL & 239 & $23 \%(20 \%-25 \%)$ \\
\hline & c. Below FPL & 219 & $21 \%(17 \%-24 \%)$ \\
\hline \multirow[t]{3}{*}{ Household Language } & a. English & 856 & $81 \%(78 \%-84 \%)$ \\
\hline & b. Spanish & 141 & $13 \%(11 \%-16 \%)$ \\
\hline & c. Other or Mix & 61 & $6 \%(4 \%-7 \%)$ \\
\hline \multirow[t]{3}{*}{ County of Residence } & a. Miami-Dade & 479 & $45 \%(44 \%-47 \%)$ \\
\hline & b. Broward & 343 & $32 \%(31 \%-34 \%)$ \\
\hline & c. Palm Beach & 236 & $22 \%(21 \%-24 \%)$ \\
\hline \multicolumn{4}{|l|}{ Access to Care Difficulties } \\
\hline \multirow[t]{2}{*}{ 1. Access difficulty finding a doctor } & a. No & 920 & $87 \%(84 \%-89 \%)$ \\
\hline & b. Yes & 138 & $13 \%(11 \%-16 \%)$ \\
\hline \multirow[t]{2}{*}{ 2. Access difficulty getting appointment } & a. No & 852 & $81 \%(78 \%-83 \%)$ \\
\hline & b. Yes & 206 & $19 \%(17 \%-22 \%)$ \\
\hline \multirow[t]{2}{*}{ 3. Access difficulty due to cost of doctor } & a. No & 916 & $87 \%(84 \%-89 \%)$ \\
\hline & b. Yes & 142 & $13 \%(11 \%-16 \%)$ \\
\hline \multirow[t]{2}{*}{ 4. Access difficulty due transportation to doctor } & a. No & 952 & $90 \%(88 \%-92 \%)$ \\
\hline & b. Yes & 106 & $10 \%(8 \%-12 \%)$ \\
\hline \multirow[t]{2}{*}{ 5. Access difficulty due to available office hours } & a. No & 860 & $81 \%(78 \%-84 \%)$ \\
\hline & b. Yes & 198 & $19 \%(16 \%-22 \%)$ \\
\hline \multirow[t]{2}{*}{ 6. Access difficulty due $\mathrm{Rx}$ cost } & a. No & 943 & $89 \%(87 \%-91 \%)$ \\
\hline & b. Yes & 116 & $11 \%(9 \%-13 \%)$ \\
\hline \multirow{2}{*}{$\begin{array}{l}\text { 7. Access difficulty due to cultural or } \\
\text { language differences }\end{array}$} & a. No & 996 & $94 \%(92 \%-96 \%)$ \\
\hline & b. Yes & 62 & $6 \%(4 \%-8 \%)$ \\
\hline
\end{tabular}

Note: Weighted estimates may not always sum to the total sample size of $\mathrm{N}=1057$ because of rounding error of the weighted estimates. CI, confidence interval; FPL, Federal poverty level; HHS, Department of Health and Human Services; NHB, non-Hispanic black; NHW, non-Hispanic white; Rx, prescription.

ED at least once in a year. ${ }^{3}$ Results from the present population-weighted survey converge with that estimate. More notably, the present study found $16 \%$ or more of children potentially visiting the ED multiple times in a year.

Although the present study found a consistent relationship between younger age of the child and greater ED utilization, common demographic and SES associations with pediatric ED visits encountered in the pediatric literature ${ }^{3-5,18}$ were not entirely consistent with these results. Specifically, there was a relatively small influence of ethnicity and poverty status on ED utilization. To some degree, this may reflect the limited information carried by the broad categorizations used in the survey. For instance, categorizations of a race and Hispanic ethnicity may wash away meaningful variability among different Hispanic groups residing in South Florida. Hispanic or Latino residents in South Florida come from a diverse range of populations comprising very different socioeconomically stratified countries of origin. ${ }^{19}$ 


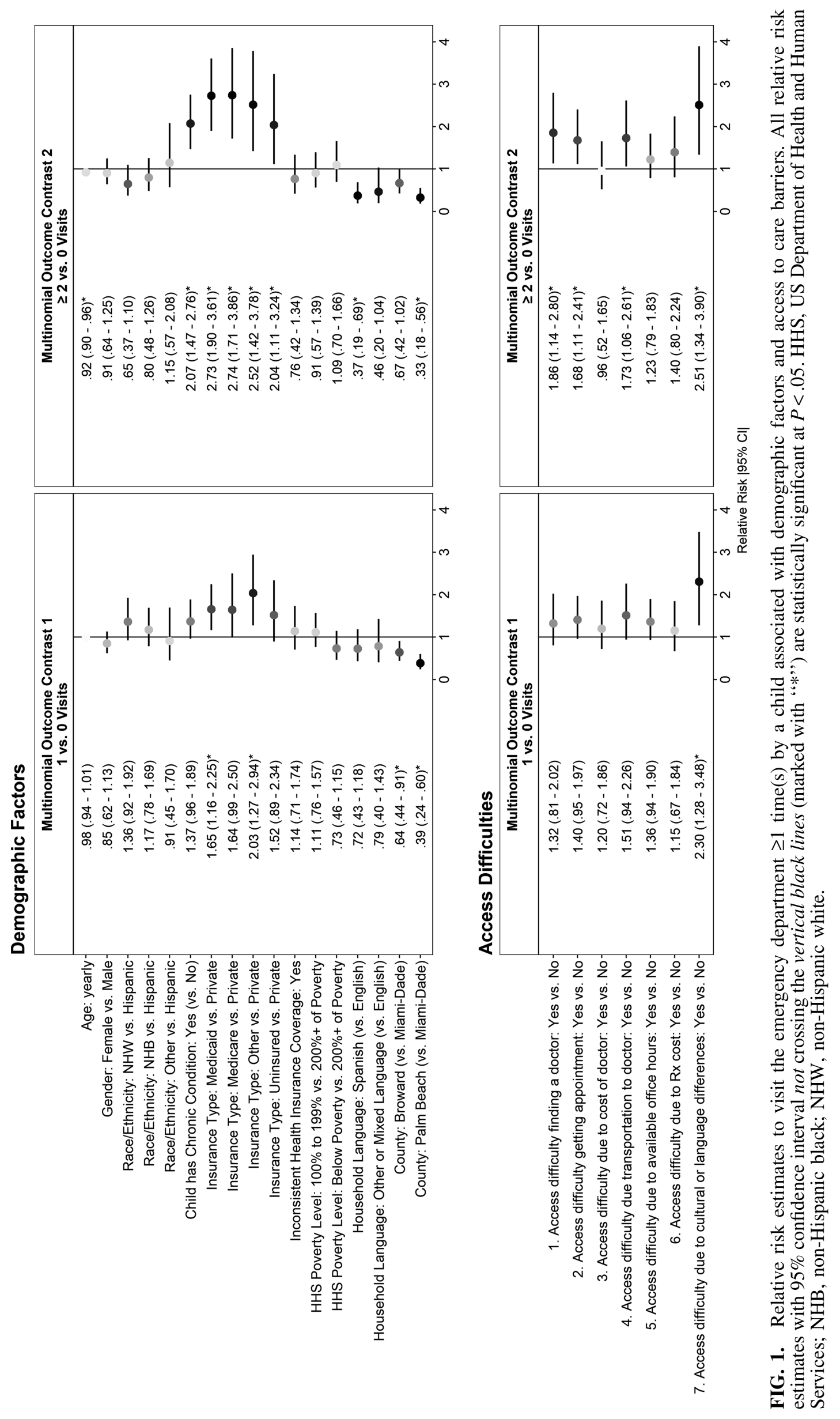




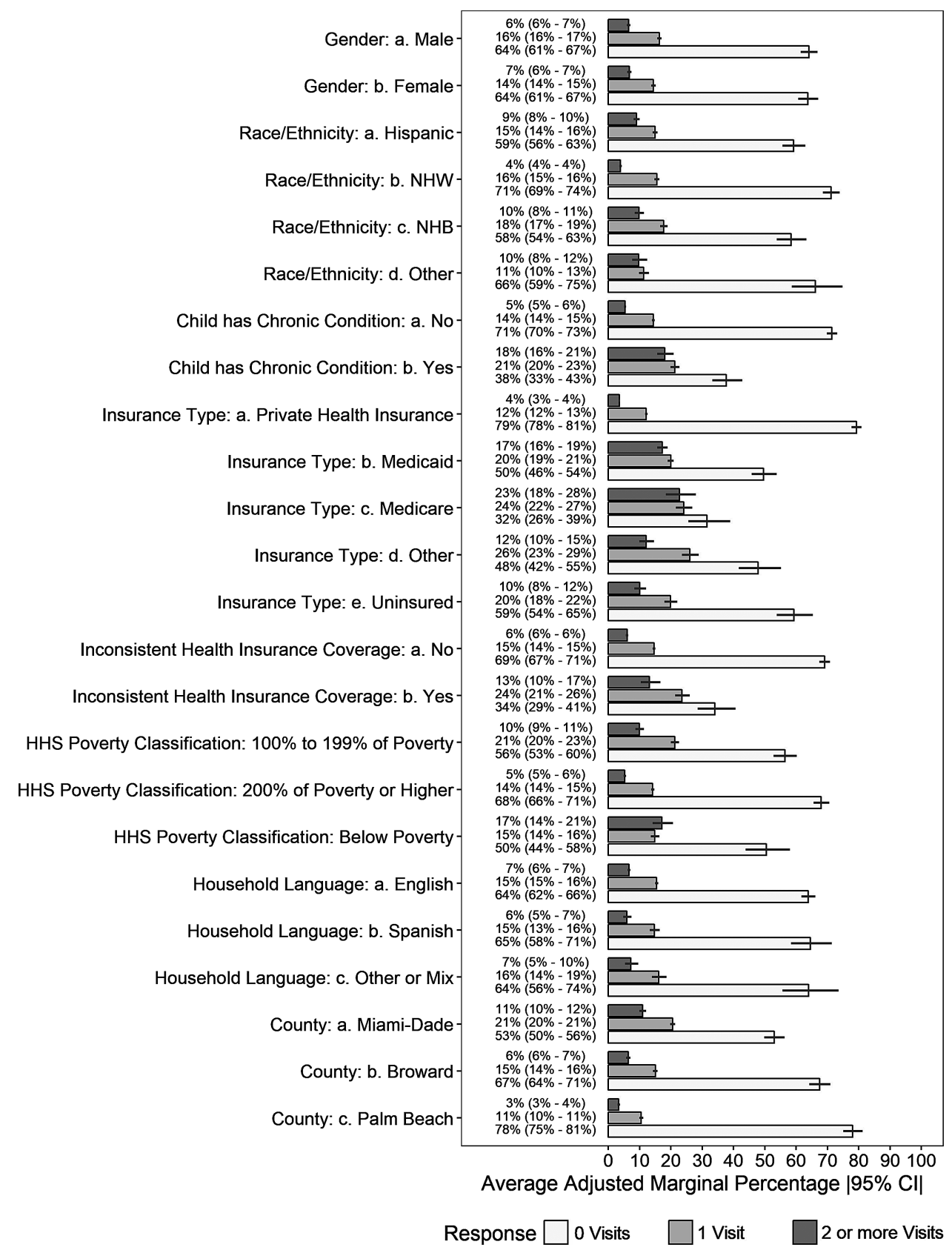

FIG. 2. Probabilities of emergency department visit frequencies by different demographic subgroups. Probabilities are presented as weighted multivariate adjusted average marginal predicted percentages for each demographic covariate included in the multinomial model predicting multiple visits $(\geq 2)$, a single visit (1), or no (0) visits in the past 12 months. Each bar represents the average marginal predicted point estimate for each level of the covariate by 3 categories of the emergency department visit frequency. Black horizontal lines represent the 95\% confidence interval (CI) of the estimate. HHS, US Department of Health and Human Services; NHB, non-Hispanic black; NHW, non-Hispanic white

Additionally, the 3-category HHS poverty status metric available to this study may have been relatively coarse in categorization of potential economic hardship. Nonetheless, this study was able to analyze a number of access to care barriers experienced by more disadvantaged groups that usually are not measured in other data sources.

At a macro level, the findings in this study further highlight the significance of access barriers related to SES. Although Miami-Dade, Broward, and Palm Beach are contiguous, Census estimates show that different subpopulations reside in each of these counties. In particular, Miami-Dade county has more recent immigrants from the Caribbean, and Central and South America than Broward or Palm Beach counties. ${ }^{19,20}$ Moreover, relative to the United States as a whole as well as Broward and Palm Beach counties, Miami-Dade county households are poorer, are living in poverty, have lower levels of educational attainment, have less health insurance coverage, have more family members living in the same residence, and also face issues associated with more dense urban environments such as longer wait times in transit. ${ }^{19,20}$ 


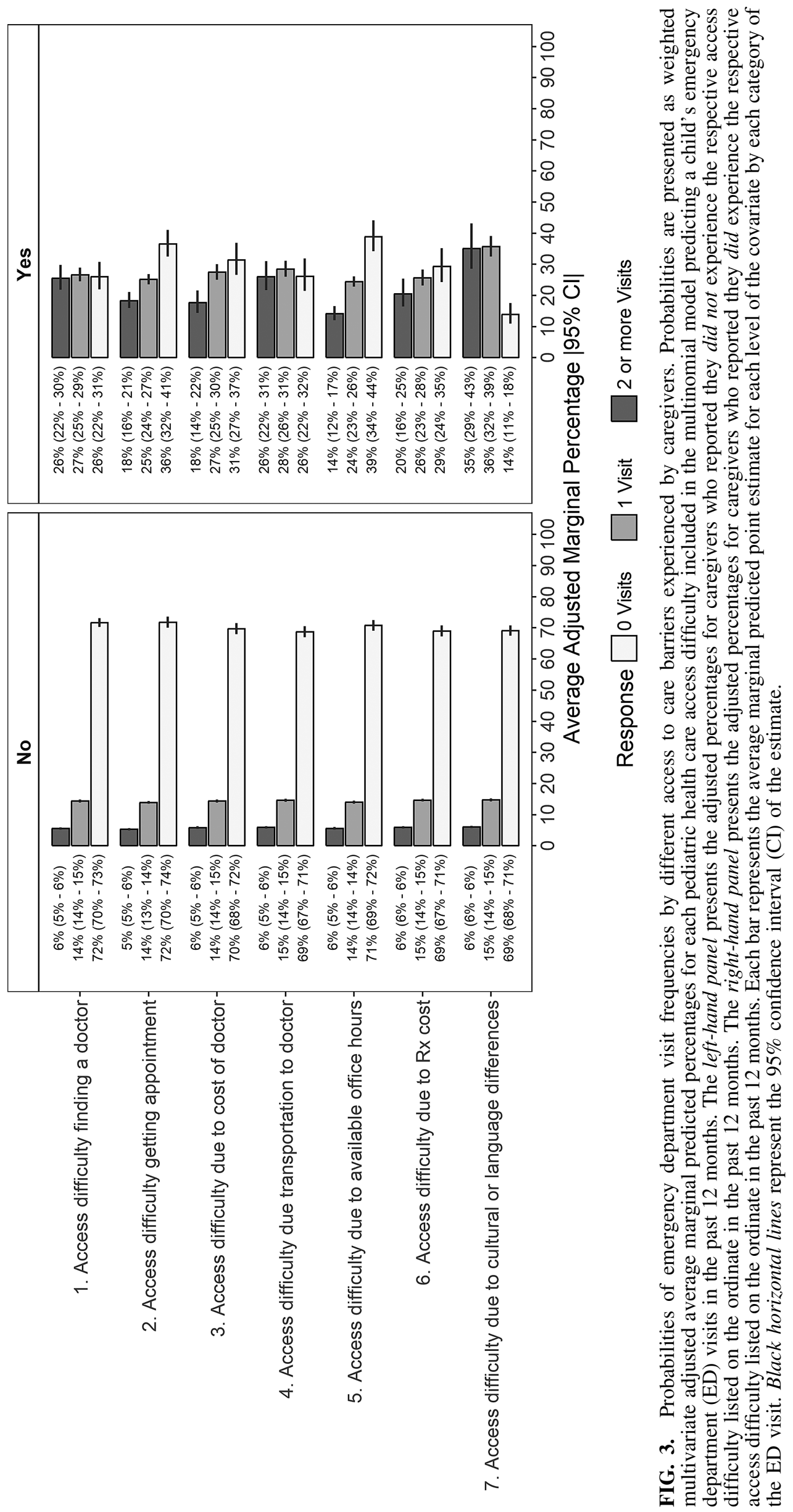


Present study findings converge on a general consideration that largely immigrant communities who may not speak English may face multiple barriers to access to care. In addition, immigration status also may play a relevant role in seeking care before an emergency arises. Specifically, evidence suggests that bureaucratic and registration systems may hinder access to timely care for undocumented immigrants, as does a general lack of familiarity with US health care systems. ${ }^{21}$

With respect to the language spoken in the household and similar acculturation dynamics, households that are less US acculturated tend to have a lower likelihood of ED visits in a given time period relative to more acculturated households. As families acculturate to US norms, their ED utilization increases. $^{22}$ One interpretation for this pattern may be that caregivers who are less acculturated may be less inclined to seek care anywhere, while those who are more acculturated find the ED a convenient place to seek care. ${ }^{23}$

At the same time, perceived convenience of the ED for care may indirectly hint at a general lack of health literacy that makes it difficult to navigate local health care systems. To the extent this interpretation is accurate, it offers an opportunity for health care-related educational interventions. ${ }^{24}$ Such educationally-oriented interventions can occur at the point of contact in the ED (ie, health care professionals) to better support patients who are likely to have primary care and specialty care follow-up needs.

Basic considerations regarding enrollment in a health insurance program may represent an opportunity for intervention as well. Consistent with much of the prior literature in both adult ${ }^{25}$ and pediatric care, ${ }^{7,8,26}$ having any form of public insurance was associated with an increased reliance on the ED. In part, this may reflect some degree of selection within which children with more medically or economically complex circumstances become eligible for public insurance options such as Medicaid, Medicare, and the Children's Health Insurance Program. Yet, some evidence suggests that those who have recently obtained health insurance coverage for themselves and their children still may retain some degree of low health literacy. ${ }^{27}$ As such, bolstering point-ofenrollment health care education may be beneficial to the caregiver, the health care institutions that may provide care to the child, and the public insurance system.

There also may be opportunities for intervention to decrease regular reliance on the ED by caregivers once an initial ED visit has occurred. For example, it is recommended that health care systems work to bolster their social work and case management support staff presence in the ED so that followup, education, and needs can be addressed more adequately at the point of care. ${ }^{28,29}$ This also may limit the time burdens faced by health care professionals when meeting with pediatric patients and their caregivers.

With respect to community support outside of the ED or hospital setting, one approach to improving care for children with chronic conditions gaining traction in recent years is an increased emphasis on promoting community health workers to support caregivers. ${ }^{30}$

The present study found that, among the access to care difficulties, cultural or language differences were most strongly related to multiple ED visits. Much evidence supports that ED utilization may increase when there is limited ability to communicate effectively with health care provid- ers. $^{31,32}$ Unfortunately, pediatric primary care may not always accommodate the language and cultural considerations of caregivers or children. ${ }^{33}$ Examples of basic components of culturally and language-conscious care include adequately listening to an individual, adequately answering questions, adequately explaining medications and their effects, and providing adequate empathy for the patient's situation. ${ }^{34}$ Doing this for both a young child and an adult may present extra challenges for providers in pediatric settings-particularly when there is a mismatch in language or health beliefs and perceptions.

In this respect, this study highlights what likely remains as an extra challenge faced by care providers in pediatric settings. Nevertheless, educational interventions at the point of care may represent opportunities for education on essential care follow-up between the provider and the child's caregiver(s). ${ }^{24,35}$ At the same time, this approach is not without significant challenges. For example, educational efforts and teach-back can be a challenge to effectively standardize ${ }^{24}$ and this speaks to the heterogeneity of health literacy needs that many caregivers may have-particularly in areas where diverse populations reside.

The potential role of narrow health care insurance networks and pediatric ED admissions also cannot be overlooked. The study team found that reports of difficulty finding a doctor for the child in the past 12 months were associated with an increased risk of multiple ED visits.

There is clear evidence that lack of available primary or specialty care for children in need tends to be associated with emergency care seeking ${ }^{36}$ and that improving access to primary care likely will diminish this behavior. ${ }^{37}$ Parallel with the importance of access to pediatric physicians overall, access to regular and convenient appointments for the child also were associated with ED visits in the past 12 months in the current study. To some degree, this may reflect a general narrowing of networks of available pediatric physicians who can meet the demand for care by the community in a timely fashion. Indeed, a mix of increased access to health insurance and a general narrowing of networks might cause demand for children's health care to outstrip available supply. ${ }^{38}$

Finally, this study found that access to care barriers attributed to transportation challenges also were associated with multiple ED visits. Here, the literature is somewhat mixed. On one hand, closer proximity to an ED is associated with a greater number of ED visits. ${ }^{3}$ On the other hand, lack of efficient and reliable transportation issues faced by many caregivers impacts their ability to bring a child to necessary care in a timely fashion. ${ }^{13,39}$ From this standpoint, health care systems could work to bolster the efficiency of other forms of care for children, such as more robust telehealth initiatives, ${ }^{8,40}$ that accommodate the language and technology device preferences of patients and caregivers.

\section{Limitations}

Although the data in this study were weighted to reflect survey nonresponse and the demographically diverse population of urban South Florida, sampling did not occur in more rural areas of South Florida. As a result, generalizability to more rural communities may be limited. It also must be acknowledged that the wording of the item in the CHNA phone interview pertaining to language and culture 
was inadvertently asked as a "double-barreled" question (ie, 2 questions asked as 1); this may have introduced ambiguity in caregiver interpretation of that survey question. In the present study it remains difficult to disentangle the specific causal mechanisms resulting in ED visits that were found embedded within cultural and language barriers that caregivers may have experienced when seeking health care for the child.

\section{Conclusions}

This representative survey found health care access barriers were associated with multiple pediatric ED visits in a 12-month period even after accounting for type of health insurance, poverty status, household language, child age, and race/ethnicity. Access barriers related to cultural and language challenges in particular may affect some caregivers' ability to seek timely care for their children. Difficulty finding a doctor and difficulty making an appointment were associated with multiple ED admissions in a year. This may reflect potential impacts of narrow networks on pediatric access to care. Additionally, caregivers may experience limited accessibility to convenient and accommodating pediatric primary care.

This could lead to an exacerbation of complications for some pediatric patients and perhaps also lead some caregivers to see the ED as the only viable option for care under their respective circumstances. However, access to care barriers measured in this study are potentially modifiable and appropriate interventions can lead to limiting unnecessary ED visits and the associated strain this places on children, caregivers, payers, and health care systems in general.

\section{Author Disclosure Statement}

The authors declare that there are no conflicts of interest The authors received no financial support for this article.

\section{References}

1. Farion KJ, Wright M, Zemek R, et al. Understanding lowacuity visits to the pediatric emergency department. PLoS One 2015;10:e0128927.

2. Kubicek K, Liu D, Beaudin C, et al. A profile of non-urgent emergency department usage in an urban pediatric hospital. Pediatr Emerg Care 2012;28:977-984.

3. Neuman MI, Alpern ER, Hall M, et al. Characteristics of recurrent utilization in pediatric emergency departments. Pediatrics 2014;134:e1025-e1031.

4. Zimmer KP, Walker A, Minkovitz CS. Epidemiology of pediatric emergency department use at an urban medical center. Pediatr Emerg Care 2005;21:84-89.

5. Alpern ER, Clark AE, Alessandrini EA, et al. Recurrent and high-frequency use of the emergency department by pediatric patients. Acad Emerg Med 2014;21:365-373.

6. Weech-Maldonado R, Morales LS, Spritzer K, Elliott M, Hays RD. Racial and ethnic differences in parents' assessments of pediatric care in Medicaid managed care. Health Serv Res 2001;36:575-594.

7. Nikpay S, Freedman S, Levy H, Buchmueller T. Effect of the Affordable Care Act Medicaid expansion on emergency department visits: evidence from state-level emergency department databases. Ann Emerg Med 2017;70:215225.e6.
8. Phelps K, Taylor C, Kimmel S, Nagel R, Klein W, Puczynski S. Factors associated with emergency department utilization for nonurgent pediatric problems. Arch Fam Med 2000;9:1086.

9. Olson LM, Tang SS, Newacheck PW. Children in the United States with discontinuous health insurance coverage. N Engl J Med 2005;353:382-391.

10. Cheng TL, Wise PH, Halfon N. Promise and perils of the Affordable Care Act for children. JAMA 2014;311:17331734.

11. Grace AM, Horn I, Hall R, Cheng TL. Children, families, and disparities: pediatric provisions in the Affordable Care Act. Pediatr Clin North Am 2015;62:1297-1311.

12. Wong CA, Kan K, Cidav Z, Nathenson R, Polsky D. Pediatric and adult physician networks in Affordable Care Act marketplace plans. Pediatrics 2017 [Epub ahead of print]; DOI: $10.1542 /$ peds.2016-3117.

13. Syed ST, Gerber BS, Sharp LK. Traveling towards disease: transportation barriers to health care access. J Community Health 2013;38:976-993.

14. Hampers LC, Cha S, Gutglass DJ, Binns HJ, Krug SE. Language barriers and resource utilization in a pediatric emergency department. Pediatrics 1999;103(6 pt 1):12531256.

15. Flores G. Culture and the patient-physician relationship: achieving cultural competency in health care. J Pediatr 2000;136:14-23.

16. Stekhoven DJ, Bühlmann P. MissForest-non-parametric missing value imputation for mixed-type data. Bioinformatics 2012;28:112-118.

17. US Department of Health and Human Services. 2015 Poverty Guidelines. 2015. https://aspe.hhs.gov/2015-povertyguidelines Accessed December 1, 2017.

18. Dy CJ, Lyman S, Do HT, Fabricant PD, Marx RG, Green DW. Socioeconomic factors are associated with frequency of repeat emergency department visits for pediatric closed fractures. J Pediatr Orthop 2014;34:548-551.

19. Motel S, Patten E. The 10 Largest Hispanic Origin Groups: Characteristics, Rankings, Top Counties. www.pewhispanic .org/files/2012/06/The-10-Largest-Hispanic-Origin-Groups.pdf Accessed July 30, 2018.

20. US Census Bureau. QuickFacts Palm Beach County, Florida; Broward County, Florida; Miami-Dade County, Florida; UNITED STATES. 2018. www.census.gov/quickfacts/ fact/table/US/PST045217 Accessed July 30, 2018.

21. Hacker K, Anies M, Folb BL, Zallman L. Barriers to health care for undocumented immigrants: a literature review. Risk Manag Healthc Policy 2015;8:175-183.

22. Allen L, Cummings J. Emergency department use among Hispanic adults: the role of acculturation. Med Care 2016; 54:449-456.

23. Nokoff N, Brunner AM, Linakis JG, Amanullah S. Presentation to either the pediatric emergency department or primary care clinic for acute illness: the caregivers' perspective. Pediatr Emerg Care 2014;30:146-150.

24. Morgan SR, Chang AM, Alqatari M, Pines JM. Nonemergency department interventions to reduce ED utilization: a systematic review. Acad Emerg Med 2013;20:969-985.

25. Zuckerman S, Shen Y-C. Characteristics of occasional and frequent emergency department users: do insurance coverage and access to care matter? Med Care 2004;42:176-182.

26. Stone ML, LaPar DJ, Mulloy DP, et al. Primary payer status is significantly associated with postoperative mortality, morbidity, and hospital resource utilization in pedi- 
atric surgical patients within the United States. J Pediatr Surg 2013;48:81-87.

27. Morrison AK, Myrvik MP, Brousseau DC, Hoffmann RG, Stanley RM. The relationship between parent health literacy and pediatric emergency department utilization: a systematic review. Acad Pediatr 2013;13:421-429.

28. Kumar GS, Klein R. Effectiveness of case management strategies in reducing emergency department visits in frequent user patient populations: a systematic review. J Emerg Med 2013;44:717-729.

29. Van den Heede K, Van de Voorde C. Interventions to reduce emergency department utilisation: a review of reviews. Health Policy Amst Neth 2016;120:1337-1349.

30. Campbell JD, Brooks M, Hosokawa P, Robinson J, Song L, Krieger J. Community health worker home visits for Medicaid-enrolled children with asthma: effects on asthma outcomes and costs. Am J Public Health 2015;105:23662372.

31. Yu SM, Huang ZJ, Schwalberg RH, Nyman RM. Parental English proficiency and children's health services access. Am J Public Health 2006;96:1449-1455.

32. Rogers AJ, Delgado CA, Simon HK. The effect of limited English proficiency on admission rates from a pediatric ED: stratification by triage acuity. Am J Emerg Med 2004;22: 534-536.

33. Seid M, Stevens GD, Varni JW. Parents' perceptions of pediatric primary care quality: effects of race/ethnicity, language, and access. Health Serv Res 2003;38:1009-1032.

34. Morales LS, Cunningham WE, Brown JA, Liu H, Hays RD. Are Latinos less satisfied with communication by health care providers? J Gen Intern Med 1999;14:409-417.
35. Sturm JJ, Hirsh D, Weselman B, Simon HK. Reconnecting patients with their primary care provider: an intervention for reducing nonurgent pediatric emergency department visits. Clin Pediatr (Phila) 2014;53:988-994.

36. Stingone JA, Claudio L. Disparities in the use of urgent health care services among asthmatic children. Ann Allergy Asthma Immunol 2006;97:244-250.

37. Cecil E, Bottle A, Cowling TE, Majeed A, Wolfe I, Saxena S. Primary care access, emergency department visits, and unplanned short hospitalizations in the UK. Pediatrics 2016;137:e20151492.

38. Institute of Medicine. Transforming Health Care Scheduling and Access: Getting to Now. Washington, DC: National Academies Press, 2015.

39. Nance ML, Carr BG, Branas CC. Access to pediatric trauma care in the United States. Arch Pediatr Adolesc Med 2009;163:512-518.

40. Keely E, Liddy C, Afkham A. Utilization, benefits, and impact of an e-consultation service across diverse specialties and primary care providers. Telemed J E Health 2013; 19:733-738.
Address correspondence to: Thom Taylor, PhD Nicklaus Children's Research Institute 701 Waterford Way, Suite 300 Miami, FL 33126

E-mail: thomas.taylor@nicklaushealth.org 